\title{
An overview of the published and running randomized phase 3 clinical results of radiotherapy in combination with immunotherapy
}

\author{
Ben G. L. Vanneste, Evert J. Van Limbergen, Kobe Reynders, Dirk De Ruysscher \\ Department of Radiation Oncology (MAASTRO Clinic), GROW - School for Oncology and Developmental Biology, Maastricht University Medical \\ Center, Maastricht, The Netherlands \\ Contributions: (I) Conception and design: BGL Vanneste; (II) Administrative support: Leonard Wee; (III) Provision of study materials or patients: \\ None; (IV) Collection and assembly of data: BGL Vanneste, EJ Van Limbergen; (V) Data analysis and interpretation: BGL Vanneste, EJ Van \\ Limbergen; (VI) Manuscript writing: All authors; (VII) Final approval of manuscript: All authors. \\ Correspondence to: Ben G. L. Vanneste. Department of Radiation Oncology (MAASTRO Clinic), GROW - School for Oncology and Developmental \\ Biology, Maastricht University Medical Center, Maastricht, The Netherlands. Email: ben.vanneste@maastro.nl.
}

\begin{abstract}
Several studies have established that radiotherapy (RT) in combination with immunotherapy (IO) has a strong synergistic effect. RT changes the tumor microenvironment, generates local inflammation reactions, and enhances immunostimulatory effects, which are able to assist IO with improving local and systemic tumor control. In several pre-clinical reports, RT in combination with IO reveals regression of tumors locally (irradiated sites) and systemically (non-irradiated sites). Several clinical trials are currently running, mostly as phase I and II studies. This article provides an overview of the randomized, prospective reported and recruiting phase 3 clinical trials of RT in combination with IO. To date, three phase 3 trials have been published on RT and sequential IO with variable results, ranging from no significant difference (Kwon et al., START) to absolute differences in overall survival of $13.5 \%$ after 3 years (PACIFIC), respectively. No phase 3 randomized trials have been published on the simultaneous combination of RT with IO. Thirty trials are presently under way, and still recruiting patients to quantify the response to RT with IO. These studies fall into three categories of research interests: (I) to discover an enhancement effect of IO as induction therapy with RT; (II) to determine the additional effect of concurrent IO on the local effect of RT; and (III) to determine the additional effect of adjuvant or consolidation IO on the local effect of RT. Most of the ongoing studies are a combination of these interests, with 15 trials evaluating the concurrent RT+IO with IO consolidation strategy. The results in coming years will provide more insights in the role of RT as an activator of the immune system, the effect of IO as local sensitizer of RT, the optimal sequencing of IO with RT, and the total RT doses needed to obtain the optimal local and systemic effect.
\end{abstract}

Keywords: Radiotherapy; immunotherapy; review; clinical phase 3 trials; radiosensitization

Submitted Feb 20, 2020. Accepted for publication Oct 28, 2020.

doi: $10.21037 /$ tlcr-20-304

View this article at: http://dx.doi.org/10.21037/tlcr-20-304

\section{Introduction}

Radiotherapy (RT) primarily functions by destroying the DNA (deoxyribonucleic acid) of irradiated cancer cells. RT also changes the tumor microenvironment, thereby producing a range of effects including local inflammatory reaction, T-cells promotion, and provoking an enhanced host immune response against tumor cells (1). These localized processes can even be improved by triggering the immune system through immunotherapy (IO) $(2,3)$. Indeed, RT induces cell damage which releases tumorspecific antigens and pro-inflammatory molecules that may produces an immunological response (i.e., immunogenic cell death), that can result in the improved priming and activation of cytotoxic T cells (e.g., dendritic and CD8 T-lymphocytes). RT further leads to the release of effector T-cell attracting chemokines and the upregulation of 


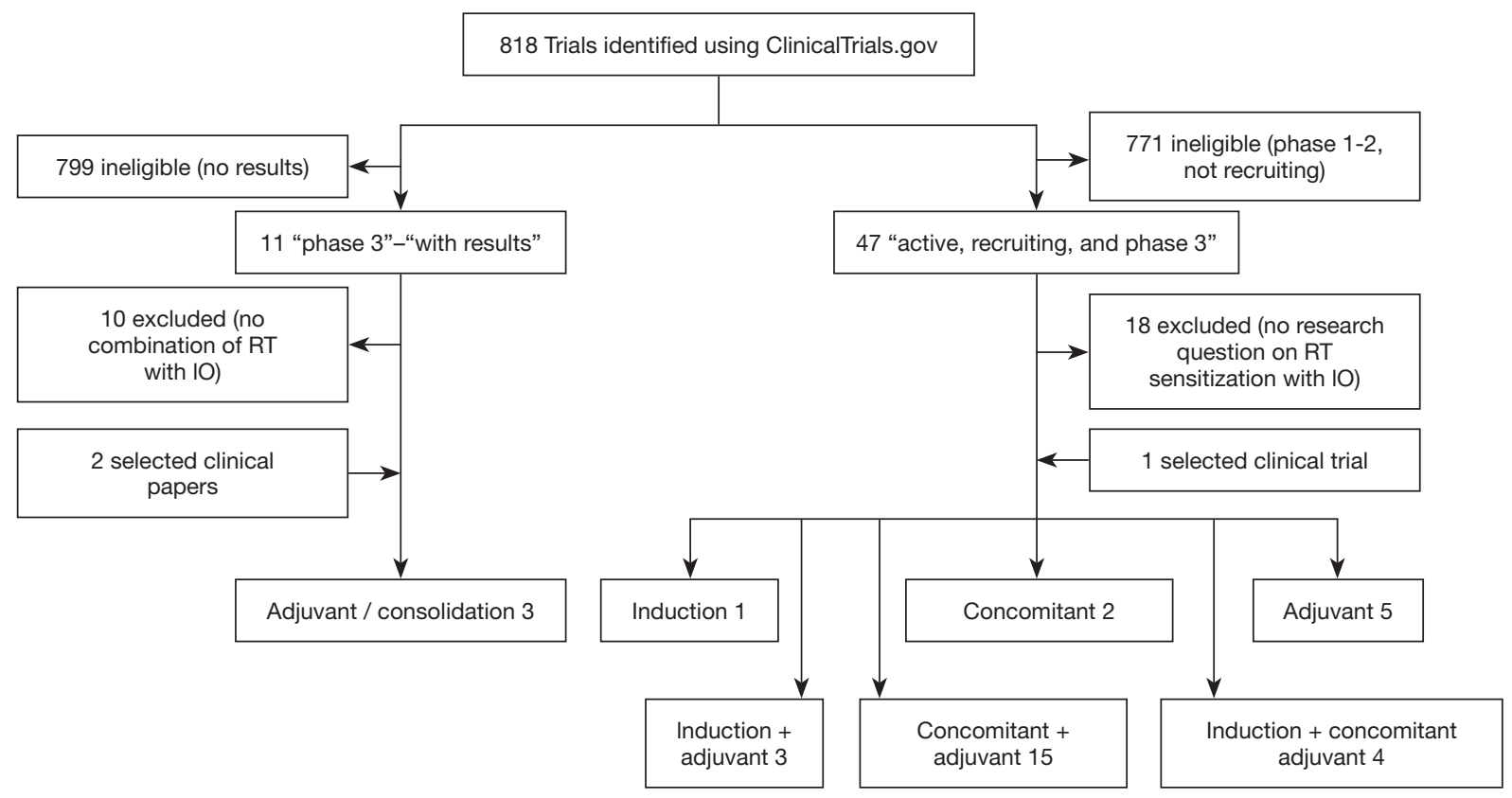

Figure 1 Flowchart of published and ongoing phase 3 randomized trials screening and including.

surface receptors which make tumor cells more vulnerable to the invasion of regulatory T-cells (Tregs) and myeloidderived suppressor cells $(3,4)$. RT is thus able to convert a partially or completely non-immunogenic tumor ('cold' tumor) into an immunogenic tumor ('hot' tumor). This unique relationship is the rationale for combining RT with IO. In this overview, we will present the reported and the (imminently) recruiting phase 3 randomized clinical trials on the combination of RT with IO.

\section{Overview of published randomized studies}

We performed a literature search in the clinicaltrials. gov database in October 2020 by using the search terms 'radiotherapy' AND 'immunotherapy', with were narrowed with the search terms 'phase 3', 'with results'. This filter revealed 11 studies, with only one reporting the combination on RT with IO (see summary in Figure 1). Additionally, two selected clinical papers from the prior knowledge of the authors have been included that met the selection criteria.

\section{Sequential radiation-IO}

Kwon and co-authors performed a double-blinded, randomized, phase 3 trial on the use of ipilimumab (a cytotoxic T-lymphocyte antigen 4, CTLA-4, inhibitor) following $1 \times 8$ gray (Gy) RT on bone metastasis in 799 prostate cancer patients with metastatic hormonal refractory status and progression after docetaxel chemotherapy (5). They found a median overall survival benefit of 11.2 months (95\% CI, 9.5-12.7) in the experimental group versus 10 months (95\% CI, 8.3-11.0) in the placebo group [hazard ratio (HR) $0.85,0.72-1.00 ; \mathrm{P}=0.053$ ]. The primary analysis revealed beneficial HR with borderline statistically significant differences in overall survival. An exploratory hazard model showed that the HR improved over time: the HR for 0 to 5 months was 1.46 (95\% CI, 1.10-1.95), for 5 to 12 months was $0.65(0.50-0.5)$, and beyond 12 months was $0.60(0.43-0.86)$. Most common grade 3-4 adverse events were immune-related and were observed in $26 \%$ of patients in the experimental group compared to $3 \%$ of patients in the placebo group. The most frequently observed grade 3-4 adverse events in the experiment group (vs. placebo) were: diarrhea 16\% (vs. 2\%), fatigue 11 (vs. $9 \%$ ), anemia $10 \%$ (vs. $11 \%$ ), and colitis $5 \%$ (vs. $0 \%$ ). One percent of deaths occurred due to toxic effects of ipilimumab. They concluded that although no statistically significant difference in overall survival was observed, there was some evidence of anti-tumor activity as measured by improved progression-free survival: 4 months (95\% CI, 3.6-4.3) with ipilimumab versus 3.1 months (2.9-3.4) with 
placebo (HR 0.70, 0.61-0.82; $\mathrm{P}<0.0001$ ).

The PACIFIC-trial was a randomized phase 3 trial $(2: 1$ ratio) showing the benefits of consolidation durvalumab (a programmed death-ligand 1 (PD-L1) inhibitor) in 713 patients with stage III unresectable non-small-cell lung cancer (NSCLC) who had completed chemoradiotherapy $(6,7)$. Both overall survival and progression free-survival were improved: the 1-, 2- and 3-year survival rates in the durvalumab group compared to the placebo group were $83.1 \%$ vs. $74.6 \%$, $66.3 \%$ vs. $55.3 \%$, and $57.0 \%$ vs. $43.5 \%$, respectively (8). The 1-year progression-free survival rate was $55.9 \%$ vs. $35.3 \%$, and the 18 -month progressionfree survival rate was $44.2 \%$ vs. $27.0 \%$. The differences remained and even increased after several updates and longer follow-up: the absolute differences in overall survival were $8.5 \%, 11 \%$, and $13.5 \%$ after respectively 1,2 , and 3 years (6-8). The median time to distant metastasis or death was 28.3 months in the experimental arm and 16.2 months in the placebo arm (HR, 0.53; 95\% CI, 0.41-0.68) (7). Furthermore, the PACIFIC-trial recorded only minor increase of toxicities: in the experimental group a total of $30.5 \%$ of the patients had grade 3 or 4 adverse events from any cause, compared to $26.1 \%$ of those in the placebo group. Of these, pneumonia was the most common side effect ( $4.4 \%$ and $3.8 \%$, respectively). Deaths due to adverse events occurred in $4.4 \%$ of subjects in the experimental arm compared to $6.4 \%$ in the placebo group.

The START-trial was a randomized phase 3 trial $(2: 1$ ratio) where 1,513 unresectable stage III NSCLC patients after concurrent chemoradiotherapy were assigned (double blinded) to tecemotide vaccine [Mucin (MUC) 1 antigenspecific] or to placebo (9). There was no significant difference in median overall survival in experimental and placebo arms: 25.6 months (95\% CI, 22.5-29.2) vs. 22.3 months (95\% CI, 19.6-25.5) respectively (adjusted HR 0.88, 0.75-1.03; $\mathrm{P}=0.123)$. Patients were stratified by timing of chemoradiotherapy as either sequential or concurrent. In the group who received previous concurrent chemoradiotherapy, the median overall survival in the tecemotide group was 30.8 months (95\% CI, 25.6-36.8) compared with the placebo group of 20.6 months $(95 \%$ CI, 17.4-23.9) $(\mathrm{P}=0.016)$. In patients treated with previous sequential chemoradiotherapy, no difference was observed in overall survival between the two groups, 19.4 versus 24.6 months, respectively $(\mathrm{P}=0.38)$. Grade $3-4$ adverse events observed with more than $2 \%$ frequency with tecemotide were: dyspnea ( $5 \%$ in the experimental group vs. $4 \%$ in the placebo group), metastases to central nervous system (3\% versus $1 \%$ ), and pneumonia (2\% versus $3 \%$ ). However, clinical development of tecemotide in lung cancer was discontinued, with a prematurely termination of the START-2 trial (NCT02049151), and of the INSPIREtrial (NCT01015443). This was the result of negative outcomes of a Japanese trial (EMR 63325-009), which was a randomized phase $1 / 2$ trial with no effect at all on every endpoint (primary endpoint: overall survival, secondary endpoints: progression-free survival, time to progression, and time to treatment failure).

\section{Concurrent radiation-IO}

To date, no phase 3 randomized trials have been reported on the simultaneous combination of RT with IO.

\section{Clinical trials in progress}

The positive results of the PACIFIC trial resulted in the introduction of IO as a standard of care in the pathway of stage III NSCLC. More IO results are becoming available, and an enormous increase in the number of trials investigating the use of IO with RT is being observed. We performed a literature search in the clinicaltrials. gov database in October 2020 by using the search terms 'radiotherapy' AND 'immunotherapy' which revealed 818 studies (Figure 1). The studies investigated different questions from RT as immune activator to IO, as sensitizer for RT, and ranging from changes in dose-fractionation to sequencing of treatment modalities. Mostly phase 1 and 2 trials were described, with a focus on the potential toxicity of combining RT with IO. The incidence and severity of pneumonitis of combined treatments was of specific interest, since both lung RT and IO can independently cause pneumonitis (10). When the search terms were narrowed to 'active, recruiting, and phase 3' trials, 47 trials were discovered. Eighteen of them were excluded because the randomization criteria did not apply to the question of either RT sensitization by IO or IO sensitization by RT. Table 1 shows the currently active phase 3 trials combining RT with IO. We divided the 30 selected trials into 3 categories of research interest: (I) studies selecting different IO as induction therapy with RT $(\mathrm{n}=1)$; (II) trials examining if RT enhances the effect of IO (concurrent) $(n=2)$; and (III) trials examining the additional effect of IO on the local effect of RT (adjuvant and consolidation) $(n=5)$. Most trials were a combination of the categories with induction and consolidation strategy in 3 trials, a 


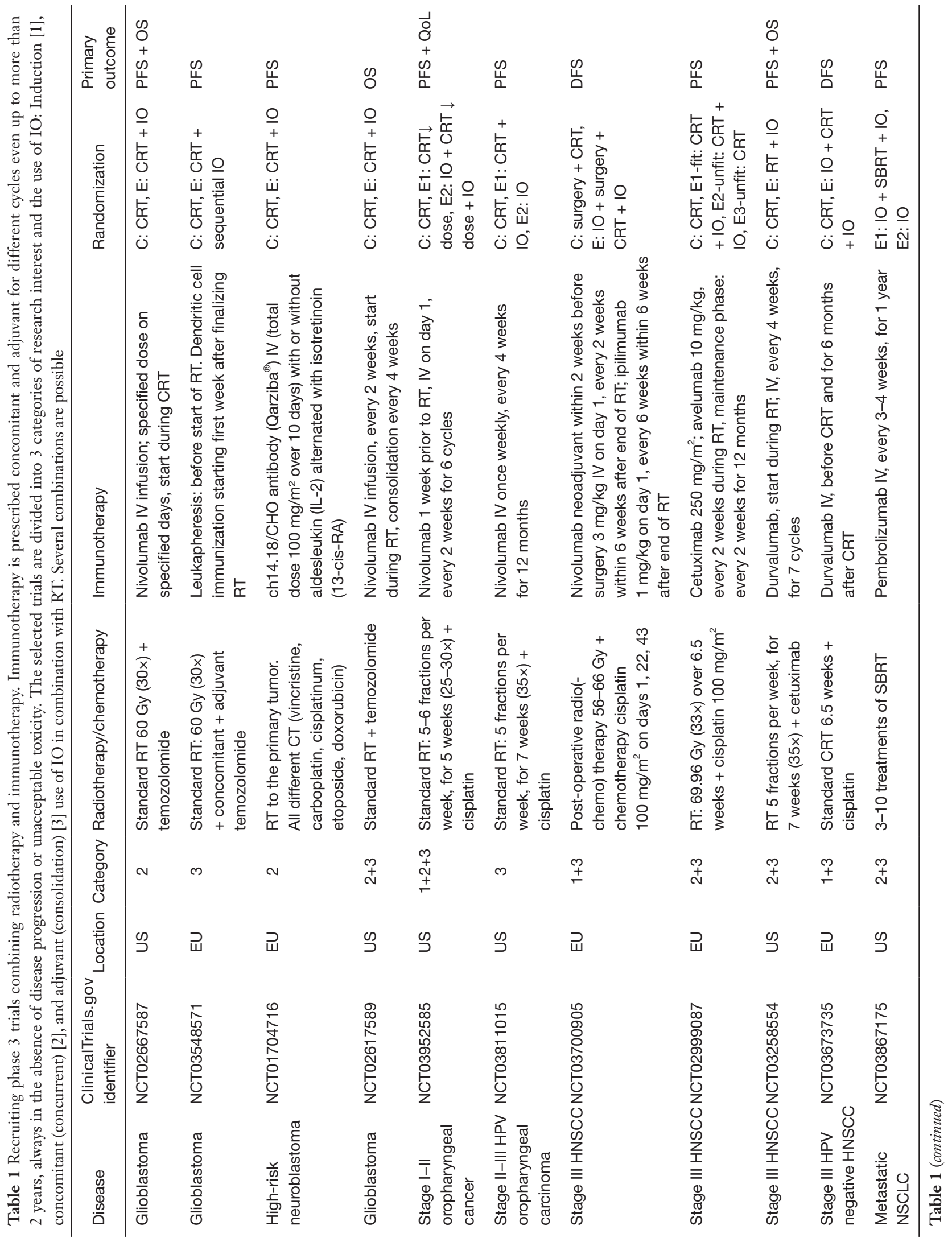




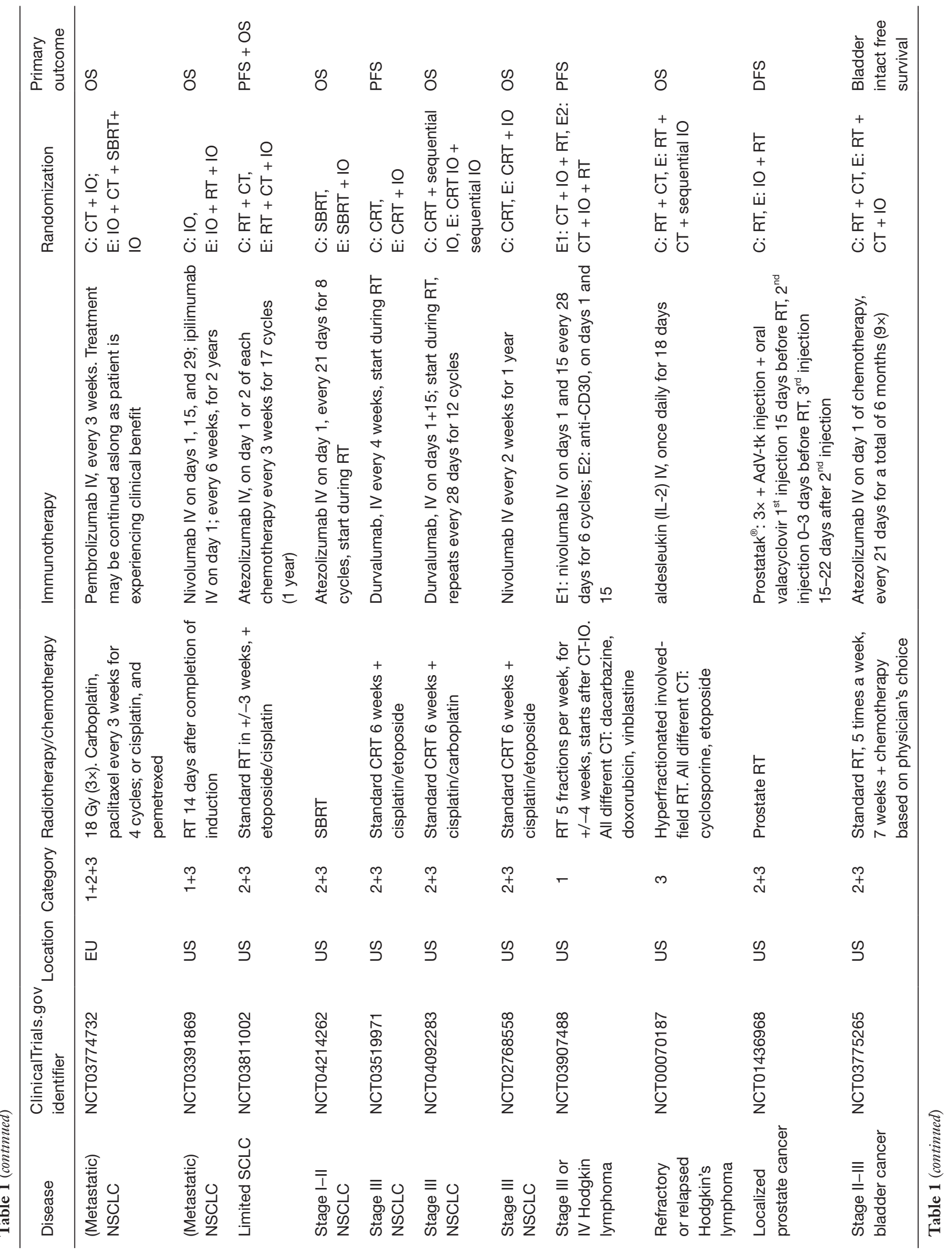




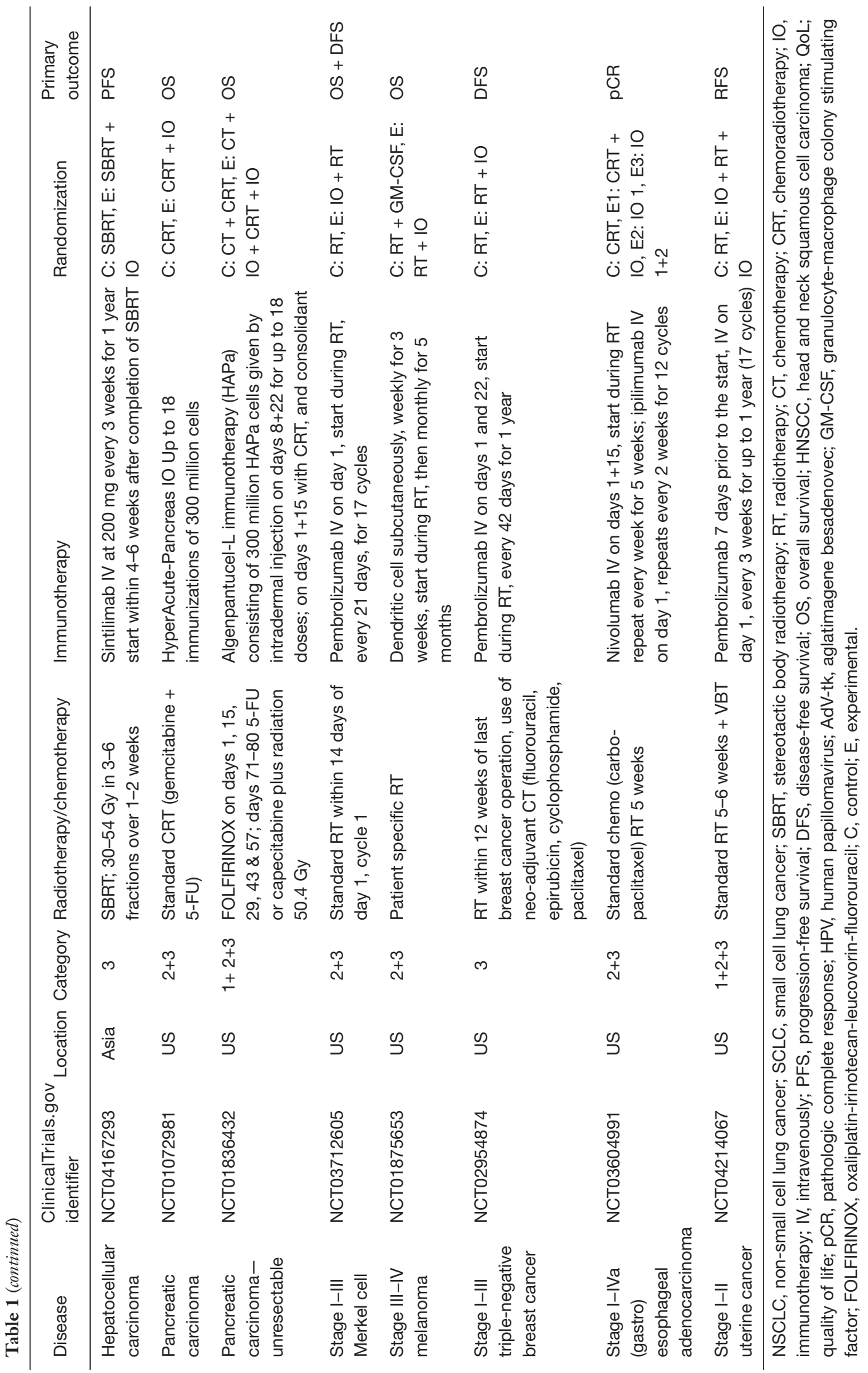


concomitant and consolidation approach in 15 trials, and all categories (induction, concomitant, and consolidation) were used in 4 trials. Several trials are presently examining the combination of chemoradiotherapy with IO $(n=20)$, where 10 trials are evaluating RT only with IO. The most used IO in the actively recruiting randomized trials were PD-1 inhibitors: nivolumab, pembrolizumab, in 9 and 5 trials, respectively. PD-L1 inhibitors as durvalumab and atezolizumab were used in 4 trials. Ipilimumab (CTLA4 inhibitor) was investigated in 3 trials. Most research was focused in the United States with 23 principal investigator centers recruiting, followed by Europe where 6 studies are recruiting. In Asia, only 1 randomized phase 3 trial was registered as running at the moment. The most researched diseases sites were lung, head and neck, and neurological cancers, which are being examined in respectively 8, 6 and 4 trials, respectively.

\section{Discussion}

Based on the preclinical and clinical work, the potential advantages of combining RT and $\mathrm{IO}$ is immense. However, most literature is either preclinical, retrospective case reports or small single-institute experiences. To date, few randomized clinical trials have been published, and not all of them were significantly positive or satisfactorily answered the predefined settings. However, all studies showed that, in the analysis of a certain subgroup, that the combination of RT with IO was effective.

Kwon and colleagues reported on the use of ipilimumab in combination with RT. Ipilimumab is a CTLA-4 inhibitor leading to a reduced CD8 T-cells and reduced Treg activity (11). This combination could synergize well with RT since Tregs lead to a suppressed immune response and tend to be more radio-resistant than other T-cells (12). However, the primary endpoint and results of this trial was negative, although the study might suggest some benefits in certain subgroups.

One of the possible reasons of not reaching the predefined thresholds (for significance), is the suboptimal sequencing of RT and IO, that is, whether the $\mathrm{IO}$ was given before or after the RT. Young and co-authors reported on the importance of sequencing (13). They revealed in preclinical data that the optimal timing of IO varied between different subclasses; anti-CTLA4 was most active when prescribed prior to RT due to regulatory $\mathrm{T}$ cell depletion. Unfortunately, in the trial of Kwon, the anti-CTLA4 was prescribed after RT. That can potentially explain the low interaction rate observed. On the other hand, other IO (anti-OX40) were ideal when prescribed one day following RT, during the post-RT period of enhanced antigen presentation. Therefore, identifying the best sequencing of RT and IO is of great significance for achieving an optimal effect.

An additional problem was, apart from the timing, that the RT dose has been shown to influence treatment efficacy. Two preclinical models revealed a comparison of different dose-fractionation regimens $(1 \times 20 \mathrm{~Gy}, 3 \times 8 \mathrm{~Gy}$, and $5 \times 6 \mathrm{~Gy})$ in combination with CTLA-4 blockade (14). They reported superiority of the multi-fraction strategy over singlefraction. The delivered dose of $1 \times 8$ Gy in the Kwon study might, unfortunately, be the wrong dose-fractionation regime.

It was noteworthy in the Kwon analysis, that the crossover of the Kaplan-Meier survival curves occurred at 7-8 months in favor of the combination therapy. This finding can be explained if patients with unfavorable profiles were faring worse, and patients with good prognostic factors survived long enough to experience the beneficial effects of the enhanced immune response. Ipilimumab was most effective in patients with low burden disease e.g. no visceral metastases, nil or minimal elevation in alkaline phosphatase levels, and no anemia. The median overall survival was in this specific group 22.7 months in the ipilimumab cohort vs. 15.8 months in the control arm $(\mathrm{P}=0.0038)$. For this reason, Beer and colleagues performed a multicenter, double-blind, phase 3 trial where minimally symptomatic or asymptomatic patients with metastatic chemotherapy-naive hormonal refractory prostate cancer were randomized to either ipilimumab or placebo (15). However, they showed no overall survival benefit; the ipilimumab cohort showed 28.7 months (95\% CI, 24.5-32.5 months) vs. 29.7 months (26.1-34.2 months) in the placebo arm (HR, 1.11; 95\% CI, $0.88-1.39 ; \mathrm{P}=0.3667)$. Median progression-free survival was 5.6 months in the experimental group $v s .3 .8$ in the control group (HR, 0.67; 95\% CI, 0.55 to 0.81 ). Notably, additional analyses revealed a higher prostate-specific antigen response rate with the experimental group (23\%) than with the control group (8\%) suggesting some tumor activity in a subgroup of patients. In this trial, no RT was given, thus no immune response could be triggered by the RT. This might explain the minimal benefits of ipilimumab as monotherapy. This is a hypothesis and should be examined in further research. Furthermore, the examined populations were different: Kwon et al. examined patients with metastatic castration-resistant prostate cancer that had 
progressed after docetaxel chemotherapy, whereas Beer et al. surveyed patients with metastatic chemotherapy-naïve castration-resistant prostate cancer.

The PACIFIC trial was a practice-changing study leading to the incorporation of durvalumab into the standard chemoradiotherapy treatment schedule of unresectable stage III NSCLC because of the continuous and even increased improved overall survival and progression free-survival within the years: the absolute differences in overall survival were increasing: $8.5 \%, 11 \%$, and $13.5 \%$ after 1,2 , and 3 years, respectively. Durvalumab is a monoclonal antibody that inhibits PD-L1 binding to PD-1 and CD80, allowing T-cells to recognize and kill tumor cells. The benefits of durvalumab were observed in both PD-L1 $>25 \%$ and $<25 \%$ subsets: prior therapy (chemotherapy and/or RT) could prime the immune system, and upregulate PD-L1, thus improving the response to subsequent IO. An exploratory analysis revealed that this benefit of durvalumab was not obvious in patients with PD-L1 expression $<1 \%$ (16). The toxicities were more or less equal: this was confirmed by comparable patient-reported quality of life scores (17).

However, some concerns can be noted:

First, prior to randomization patients were selected if no progression after initial chemoradiotherapy occurred, which is a selection bias that can explain the good outcome. Further, the total radiation dose given in the PACIFIC trial was not uniform: the schedules varied from below 54 Gy up to $74 \mathrm{~Gy}$, although the majority of patients $(92.9 \%)$ received a dose between 54 and 66 Gy. This uneven distribution in doses, that even deviated from the inclusion criteria, was based on individual dose prescription of the radiation oncologist to fulfill the criteria of the mean dose to the lung being lower than $20 \mathrm{~Gy}$, and the V20 (the volume of lung parenchyma that received 20 Gy or more) should be lower than $35 \%$, or both.

Thirdly, the time interval of administering the first durvalumab was very different between these patients, varying over 1 to 42 days, with an initial interval of 1 to 14 days. Longer intervals seemed to correlate with worse outcome. These observations are in line with preclinical studies showing best effects of PD-L1 inhibitors when radiotherapy (RT) was delivered concurrent of immediately before with short interval (18).

Alternatively, it could also be that patients experience more problems and/or toxicity during standard treatment, indicating both a tendency for worse prognosis and delay of immune treatment.

Lastly, several patients received induction chemotherapy
(26.8\%), and the concurrent chemotherapy was heterogeneous, including combinations of platinum derivate with etoposide, vinblastine, vinorelbine, paclitaxel docetaxel, or pemetrexed.

Despite these differences, the PACIFIC study was able to demonstrate the long-term clinical benefits with adjuvant durvalumab for unresectable stage III NSCLC.

The START-trial reported on the use of Tecemotide: a MUC-1 antigen specific IO capable of inducing a T-cell response to MUC-1, which is involved in interactions with tyrosine kinases and other cell surface receptors. These interactions trigger inappropriate activation of intracellular signaling pathways and promote the growth, proliferation, and survival of cancer.

The START-trial revealed no added value with respect to overall survival in stage III NSCLC patients with at least stable disease following chemoradiotherapy. However, in a subgroup analysis, there seemed to be a potential added benefit in the subgroup treated with concurrent chemoradiotherapy. The median survival in the placebo group of concurrent chemoradiotherapy was lower than was described in similar trials: patients who received standarddose chemoradiotherapy in the RTOG 0617 trial showed an overall survival of 28.7 months (95\% CI, 24.1-36.9) in comparison with 20.6 months in the START-trial population (95\% CI, 17.4-23.9) (19,20). These differences could be explained by the lower routine of PET staging in the START-trial.

This trial also had several limitations. First, the randomization of these patients again followed after successfully completing chemoradiotherapy when no progression occurred, which was a selection bias for good outcome. Secondly, there was no standardization on either radiation schedules or doses, with only a minimum limit to total dose of $50 \mathrm{~Gy}$. Moreover, quality assurance of RT was not performed, with only a verification of source data. Next, the delivered chemotherapy was heterogeneous, including combination of platinum derivate with etoposide, vinorelbine, taxanes, gemcitabine or others. Fifth, at the time that the trial was designed, little information was available about survival in these patients. Therefore, an estimation of 20 months was made for the placebo group. Finally, interpretation of the outcome was affected by the results of the clinical hold. There were patients in followup longer than planned, while others had a relatively short follow-up period from 9 months (9).

In the current research area of IO and RT, a wide range of aspects remain unknown and are now the focus of the 
current research.

First, RT can give a boost for the immune system, where randomization is between $\mathrm{IO}$ and $\mathrm{IO}$ with $\mathrm{RT}$, so as to enhance the activator role of RT on the immune system. This is examined in several trials in mainly (metastatic) lung cancer (NCT03867175, NCT03774732, NCT03391869), in oropharyngeal carcinoma (NCT03811015) and in esophageal cancer (NCT03604991). The given immunotherapies are nivolumab, pembrolizumab, and atezolizumab, in 4 trials, 2 trials, and 1 trial, respectively. RT delivered to the tumor impacts both tumor cells and surrounding stromal cells. RT-induced cancer cell damage exposes tumor-specific antigens which unmask tumors to the immune system through a process called immunogenic cell death. In turn, this process leads to improved priming and activation of cytotoxic $\mathrm{T}$ cells. RT-induced modulation of the tumor microenvironment may also facilitate the recruitment and infiltration of immune cells.

Secondly, IO can also function as a sensitizer of local RT, where randomization is between RT and RT with IO. This is examined in head and neck cancer (NCT03258554) where traditional chemoradiotherapy is compared with RT with IO. Additionally, this approach is evaluated in lung cancer (NCT04214262), prostate carcinoma (NCT01436968), Merkel cell (NCT03712605), melanoma (NCT01875653), and uterine cancer (NCT04214067). This strategy was recently reported in a review of preclinical series: Vanneste et al. calculated a median radiation enhancement factor of different IO ranging of 1.7 to 9.1 in comparison to traditionally chemotherapy of 1.1 (21). The authors concluded that for the same RT dose, a higher local control can be achieved with a combination of IO and RT in preclinical settings. This supports the use of combination therapy to improve local tumor control in clinical settings without exacerbation of toxicities. Further research has to be performed to confirm this hypothesis.

Thirdly, an additional important factor is the sequencing of the combination therapy. Several studies are evaluating IO before, during or after RT, also in combinations of concomitant with consolidation, or induction with adjuvant or even sequencing all approaches together. The ideal timing is still unclear, and all combinations are under examination in different trials.

Fourth, the optimal total RT dose and fractionation schedule is unclear. An interesting randomized phase 2 study stimulates continuing exploration in this line of research: the PEMBRO-RT trial randomized 92 advanced NSCLC patients, with unknown PD-L1 status, to receive pembrolizumab every 3 weeks either alone (control group) or after $3 \times 8$ Gy RT to a single tumor site (22). They concluded that the studies pre-stated end-point criteria were not achieved although the overall response rate was doubled. They observed the largest advantage from the RT in patients with initially PD-L1 negative tumors. This study supports the hypothesis of turning an immune 'cold' tumor into an immune 'hot' tumor: this trial clearly identified a patient subgroup (the PD-L1 negative tumors) that appears to benefit most from the RT to change the tumor microenvironment and thus enhancing the effects of pembrolizumab. The positive results were highly distorted by the PD-L1 negative subgroup, which had significantly increased progression-free survival and overall survival. They concluded that a larger study would be necessarily to define whether SBRT activates the host immune system thus improving the outcome of IO for stage IV NSCLC patients, which is now examined in different studies (NCT04214262, NCT03519971, NCT04092283, NCT02768558).

Fifth, there are different aspects that deserve greater attention: there has been no examination comparing different irradiation techniques, or on treatment areas. There are now, in the current phase 3 trials, attempts to distinguish the role of decreased lymphopenia: Chen and colleagues demonstrated that SBRT may better preserve lymphocytes (and hence improve outcomes) than traditional RT (23). Further research is needed to confirm this.

Finally, the selection of which target that should be irradiated in the context of creating an abscopal effect together with IO is not clear (24). Some authors proposed a mathematical model to predict the lesions with the highest potential. This model was based on T-cell trafficking and the assumption that abscopal effects can only be achieved when activated $\mathrm{T}$ cells from the irradiated tumor can reach the distant sites in sufficient numbers.

IO related adverse events are typically transient, but occasionally very severe. Common adverse events are dermatological reaction, diarrhea/colitis, hepatotoxicity, and endocrinopathies, although other sites can also be affected, such as nephritic, pancreatic, neurological, cardiovascular, and muscular. A combination of IO with RT shows an encouraging safety profile: no extreme increase of high-grade toxicities have been reported. However, most studies are phase 1 trials, but they do provide additional information about the possible safety profile of concomitant IO with RT. Amin and colleagues reported that concurrent RT with IO is well tolerated, with no increase of the 
toxicity profile from the previously reported toxicity rates from IO or RT alone (25). Also, the PACIFIC trial revealed equal toxicity levels. However, future research will have to confirm these findings, and cautions about counseling patients on the potentially increased rates of side effects from combination therapy is still warranted.

We foresee that combination strategies of IO with RT will further incorporated into the clinical pathway, leading to more organ preservation approaches, with even lower radiation doses, and consequently lower toxicities. This can even form the basis for future therapeutic options, and can give radiation oncologists the opportunity to play a more prominent role within the systemic treatment approach for cancer patients. Future research will be needed to confirm these ambitions.

\section{Conclusions}

The potential combination of RT and IO could become a paradigm shift in the field of oncology. However, understanding the interactions of RT with the initiated immune responses and IO remains an important challenge for future research. Well-designed randomized, clinical trials are underway to prove the benefits of these combination therapy, and to make local RT as a possible part of systemic therapy. The following years of clinical research will indeed be challenging and yet extremely important to find the optimal combination of RT and IO.

\section{Acknowledgments}

Funding: Varian.

\section{Footnote}

Provenance and Peer Review: This article was commissioned by the Guest Editors (Jacek Jassem and Rafal Dziadziuszko) for the series "Radiotherapy in thoracic malignancies" published in Translational Lung Cancer Research. The article has undergone external peer review.

Conflicts of Interest: All authors have completed the ICMJE uniform disclosure form (available at http://dx.doi. org/10.21037/tlcr-20-304). The series "Radiotherapy in thoracic malignancies" was commissioned by the editorial office without any funding or sponsorship. Dr. De Ruysscher reports grants from Brsitol-Myers Squibb, grants from AstraZeneca, grants from Boehringer Ingelheim, from
Philips, from Olink, from Celgene, from Seattle Genetics, from Roche/Genentech, from Merck/Pfizer, outside the submitted work. The authors have no other conflicts of interest to declare.

Ethical Statement: The authors are accountable for all aspects of the work in ensuring that questions related to the accuracy or integrity of any part of the work are appropriately investigated and resolved.

Open Access Statement: This is an Open Access article distributed in accordance with the Creative Commons Attribution-NonCommercial-NoDerivs 4.0 International License (CC BY-NC-ND 4.0), which permits the noncommercial replication and distribution of the article with the strict proviso that no changes or edits are made and the original work is properly cited (including links to both the formal publication through the relevant DOI and the license). See: https://creativecommons.org/licenses/by-nc-nd/4.0/.

\section{References}

1. Van Limbergen EJ, De Ruysscher DK, Olivo Pimentel $\mathrm{V}$, et al. Combining radiotherapy with Immunotherapy: the past, the present and the future. Br J Radiol 2017;90:20170157.

2. Reynders K, Illidge T, Siva S, et al. The abscopal effect of local radiotherapy: using Immunotherapy to make a rare event clinically relevant. Cancer Treat Rev 2015;41:503-10.

3. Demaria S, Coleman CN, Formenti SC. Radiotherapy: Changing the Game in Immunotherapy. Trends Cancer 2016;2:286-94.

4. De Ruysscher D. Radiotherapy and PD-L1 inhibition in metastatic NSCLC. Lancet Oncol 2017;18:840-2.

5. Kwon ED, Drake CG, Scher HI, et al. CA184-043 Investigators. Ipilimumab versus placebo after radiotherapy in patients with metastatic castration-resistant prostate cancerthat had progressed after docetaxel chemotherapy (CA184-043): a multicentre, randomised, double-blind, phase 3 trial. Lancet Oncol 2014;15:700-12.

6. Antonia SJ, Villegas A, Daniel D, et al. Durvalumab after Chemoradiotherapy in Stage III Non-Small-Cell Lung Cancer. N Engl J Med 2017;377:1919-29.

7. Antonia SJ, Villegas A, Daniel D, V et al. Overall Survival with Durvalumab after Chemoradiotherapy in Stage III NSCLC. N Engl J Med 2018;379:2342-50.

8. Gray JE, Villegas A, Daniel D, et al. Three-Year Overall Survival with Durvalumab after Chemoradiotherapy in 
Stage III NSCLC-Update from PACIFIC. J Thorac Oncol 2020;15:288-93.

9. Butts C, Socinski MA, Mitchell PL, et al. Tecemotide (L-BLP25) versus placebo after chemoradiotherapy for stage III non-small-cell lung cancer (START): a randomised, double-blind, phase 3 trial. Lancet Oncol 2014;15:59-68.

10. Bhalla N, Brooker R, Brada M. Combining immunotherapy and radiotherapy in lung cancer. J Thorac Dis 2018;10:S1447-S1460.

11. Rudd CE. CTLA-4 co-receptor impacts on the function of Treg and CD8+ T-cell subsets. Eur J Immunol 2009;39:687-90.

12. Kachikwu EL, Iwamoto KS, Liao YP, et al. Radiation enhances regulatory $\mathrm{T}$ cell representation. Int $\mathrm{J}$ Radiat Oncol Biol Phys 2011;81:1128-35.

13. Young KH, Baird JR, Savage T, et al. Optimizing Timing of Immunotherapy Improves Control of Tumors by Hypofractionated Radiation Therapy. PLoS One 2016;11:e0157164.

14. Dewan MZ, Galloway AE, Kawashima N, et al. Fractionated but not single-dose radiotherapy induces an immune-mediated abscopal effect when combined with anti-CTLA-4 antibody. Clin Cancer Res 2009;15:5379-88.

15. Beer TM, Kwon ED, Drake CG, et al. Randomized, Double-Blind, Phase III Trial of Ipilimumab Versus Placebo in Asymptomatic or Minimally Symptomatic Patients With Metastatic Chemotherapy-Naive Castration-Resistant Prostate Cancer. J Clin Oncol 2017;35:40-47

16. Paz-Ares L, Spira A, Raben D, et al. Outcomes with durvalumab by tumour PD-L1 expression in unresectable, stage III non-small-cell lung cancer in the PACIFIC trial. Ann Oncol 2020;31:798-806.

17. Hui R, Özgüroğlu M, Villegas A, et al. Patient-reported outcomes with durvalumab after chemoradiotherapy

Cite this article as: Vanneste BGL, Van Limbergen EJ, Reynders K, De Ruysscher D. An overview of the published and running randomized phase 3 clinical results of radiotherapy in combination with immunotherapy. Transl Lung Cancer Res 2021;10(4):2048-2058. doi: 10.21037/tlcr-20-304 in stage III, unresectable non-small-cell lung cancer (PACIFIC): a randomised, controlled, phase 3 study. Lancet Oncol 2019;20:1670-80

18. Dovedi SJ, Adlard AL, Lipowska-Bhalla G, et al. Acquired resistance to fractionated radiotherapy can be overcome by concurrent PD-L1 blockade. Cancer Res 2014;74:5458-68.

19. Bradley JD, Paulus R, Komaki R, et al. Standard-dose versus high-dose conformal radiotherapy with concurrent and consolidation carboplatin plus paclitaxel with or without cetuximab for patients with stage IIIA or IIIB non-smallcell lung cancer (RTOG 0617): a randomised, two-by-two factorial phase 3 study. Lancet Oncol 2015;16:187-99.

20. Bogart JA, Gajra A. Immunotherapy in non-small-cell lung cancer: a good start? Lancet Oncol 2014;15:5-6.

21. Vanneste BGL, Van Limbergen EJ, Dubois L, et al. Immunotherapy as sensitizer for local radiotherapy. Oncoimmunology 2020;9:1832760.

22. Theelen WSME, Peulen HMU, Lalezari F, et al. Effect of Pembrolizumab After Stereotactic Body Radiotherapy vs Pembrolizumab Alone on Tumor Response in Patients With Advanced Non-Small Cell Lung Cancer: Results of the PEMBRO-RT Phase 2 Randomized Clinical Trial. JAMA Oncol 2019;5:1276-82.

23. Chen D, Patel RR, Verma V, et al. Interaction between lymphopenia, radiotherapy technique, dosimetry, and survival outcomes in lung cancer patients receiving combined immunotherapy and radiotherapy. Radiother Oncol 2020;150:114-20.

24. Poleszczuk JT, Luddy KA, Prokopiou S, et al. Abscopal Benefits of Localized Radiotherapy Depend on Activated T-cell Trafficking and Distribution between Metastatic Lesions. Cancer Res 2016;76:1009-18.

25. Amin NP, Zainib M, Parker SM, et al. Multi-institutional report on toxicities of concurrent nivolumab and radiation therapy. Adv Radiat Oncol 2018;3:399-404. 